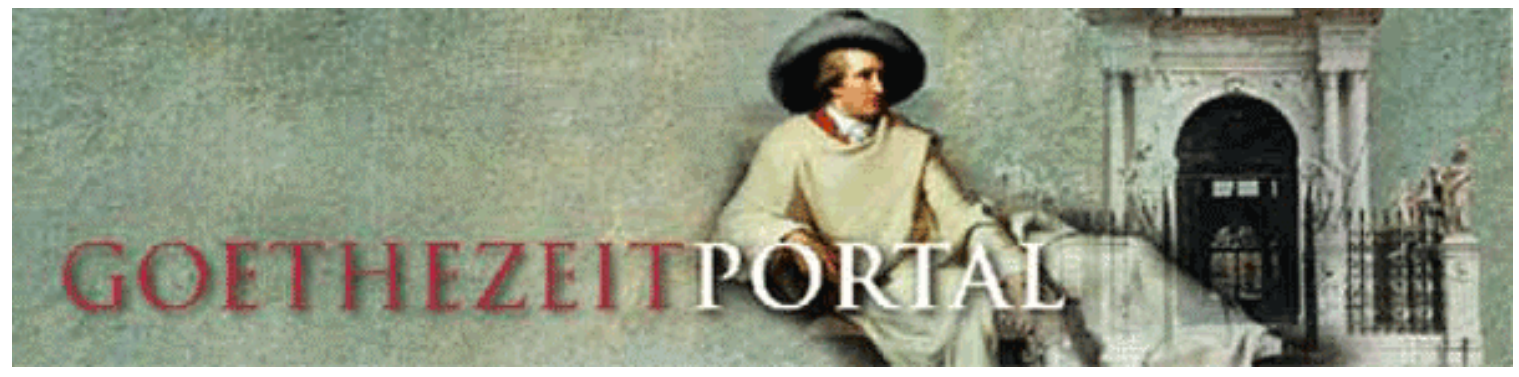

MARTIN HUBER

\title{
Der Text als Bühne. \\ Zu Jean Pauls Leben des vergnügten Schulmeisterlein \\ Maria Wutz
}

\begin{abstract}
Vorblatt
\section{Publikation}

Erstpublikation in: Schnittpunkt Romantik. Text- und Quellenstudien zur deutschen Literatur des 18. und 19. Jahrhunderts. Festschrift für Sybille von Steinsdorff. Hg. Von W. Bunzel, K. Feilchenfeldt und W. Schmitz. Tübingen: Niemeyer 1997, S. 23-35.

Vorlage: Datei des Autors

URL: < http://www.goethezeitportal.de/db/wiss/jeanpaul/wutz_huber.pdf>

Eingestellt am 11.06.2004
\end{abstract}

\section{Autor}

Prof. Dr. Martin Huber

FernUniversität in Hagen

Institut für Neuere deutsche und europäische Literatur

Universitätsstraße 11

D-58084 Hagen

Emailadresse: <martin.huber@fernuni-hagen.de>

\section{Empfohlene Zitierweise}

Beim Zitieren empfehlen wir hinter den Titel das Datum der Einstellung oder des letzten Updates und nach der URL-Angabe das Datum Ihres letzten Besuchs dieser Online-Adresse anzugeben:

Martin Huber: Der Text als Bühne. Zu Jean Pauls Leben des vergnügten Schulmeisterlein Maria Wutz (11.06.2004). In: Goethezeitportal. URL:

<http://www.goethezeitportal.de/db/wiss/jeanpaul/wutz_huber.pdf>

(Datum Ihres letzten Besuches). 


\section{MARTIN HUBER}

\section{Der Text als Bühne. \\ Zu Jean Pauls Leben des vergnügten Schulmeisterlein Maria Wutz}

Die liebenswerte Figur des Schulmeisterlein Wutz betrat 1793 im Anhang der Unsichtbaren Loge die Bühne der literarischen Öffentlichkeit. Während die zietgenössische Leserschaft den Wutz allerdings kaum zur Kenntnis nahm, wurde das Schulmeisterlein zusammen mit Quintus Fixlein nach Jean Pauls Tod 1825 zu einer der Schlüsselfiguren, in der das 19. Jahrhundert in konsequenter Überblendung von Autor, Erzähler und literarischer Figur sein Bild vom humoristischen Idylliker Jean Paul uns dessen „gemütvoll idyllische[m] Wesen“1 fand. „Die reizende Idylle: Leben des vergnügten Schulmeisterlein Wutz in Auenthal [stellt] ein abgeschlossenes Bild heitern Frohsinns in den ärmlichen Verhältnissen [dar]“ lesen wir 1879 in der Deutschen Literaturgeschichte von Robert Koenig. „Diese kleine humoristische Dichtung war aus seinen eigenen Erfahrungen herausgewachsen - sie stellte, wie er selbst sagte, $>$ das Volksglück [!] in der Beschränkung $<$ dar “2

Koenigs Literaturgeschichte reklamiert im Vorwort freilich dezidiert keinen „streng wissenschaftlichen Charakter“, sondern war vielmehr „Dem deutschen Hause“ zugedacht. Diese Perspektive mag auch die Einstellung von Jean Pauls eigener Idyllentheorie ${ }^{3}$ geschuldet sein; dennoch ist dies eine signifikante Entstellung, die nicht zufällig den Kern eines Jean Paul-Bildes trifft, wie es sich - in Literaturgeschichten und Anthologien des gebildeten Bürgertums multipliziert - weithin erhalten sollte. ${ }^{4}$ Und dies trotz Nietzsches gerade auch auf den Idyllenschreiber zielenden vernichtenden Urteils, Jean Paul sei

\footnotetext{
${ }^{1}$ So Hermann Hettner in seiner Geschichte der deutschen Literatur im achtzehnten Jahrhundert (1870); zitiert nach: Jean Paul im Urteil seiner Kritiker. Dokumente zur Wirkungsgeschichte Jean Pauls in Deutschland. Hrsg. Und eingeleitet und kommentiert von Peter Sprengel. München: Beck 1980 (= Wirkung der Literatur, Bd. 6), S.208. Hier auch weitere einschlägige Dokumente von Gottfried Gervinus, Wilhelm Heinrich Riehl und Julian Schmidt. ${ }^{2}$ Robert Koenig: Deutsche Literaturgeschichte. Bielefeld / Leipzig: Velhagen \&Klasing 1879, S. $405 f$.

${ }^{3}$ Vgl. Jean Pauls Vorschule der Ästhetik (1804), § 73 „Die Idylle“: „Wenigstens eine kleine epische Gattung haben wir, nämlich die Idylle. Diese ist nämlich epische Darstellung des Vollglücks in der Beschränkung“; zitiert nach: Jean Paul. Werke. Hrsg. Von Norbert Miller. Bd.5. München/Wien: Hanser 1987, S. 258

${ }^{4}$ Vgl. etwa Benno von Wieses Rede zum 10jährigen Bestehen der Jean-Paul-Gesellschaft am 26. Oktober 1935 in Bayreuth „Jean Paul als Dichter des deutschen Volkstums“. In: Jean Paul im Urteil seiner Kritiker (wie Anm. 1), S. 273-279.
} 
„ein Verhängnis im Schlafrock“5 . Das Bild vom Idylliker Jean Paul begann sich (zumindest in der Wissenschaft) nachhaltig erst mit Arbeiten zu wandeln, die - anknüpfend an produktionsästhetisch interessierte Studien zu Jean Paul als Erzähler ${ }^{6}$ zu Beginn der 60er Jahre unseres Jahrhunderts - erstmals das gesamte Spektrum von Jean Pauls Idyllik in den Blick nahmen. Das hieß für den Wutz, dass auch der Untertitel Eine Art Idylle ernstgenommen wurde. Mit Blick auf Gattungstheorie und Publikumserwartungen führte das zu einer Lesart als „gefährdete“ oder „gestörte Idylle“ ${ }^{\text {“7 }}$ und eröffnete schließlich Zugänge über Erzähler-Leser-Kommunikation, Geschichtlichkeit und Metaphorik des Textes ${ }^{8}$. Meine Überlegungen zu Jean Pauls Wutz nehmen diese Arbeiten auf, fragen aber ausgehend von bislang unbeobachteten kompositorischen Problemen, die der Text selbst aufwirft, nach dem Aufbau seiner narrativen Strukturen und deren Funktionen für Jean Pauls Erzählen.

${ }^{5} 1880$ in Der Wanderer und sein Schatten; Friedrich Nietzsche: Werke. Kritische Gesamtausgabe. Hrsg. Von Giorgio Colli und Mazzino Montinari. Abt.4. Bd.3. Berlin: de Gruyter 1967, s. 234f., hier: S. 235

${ }^{6}$ Etwa Walter Rehm: Jean Pauls vergnügtes Notenleben oder Notenmacher und Notenleser. In: Jahrbuch der deutschen Schillergesellschaft 3 (1959), S. 244-337); sowie Wolfdietrich Rasch: Die Erzählweise Jean Pauls. Metaphernspiele und dissonante Strukturen. München: Hanser 1961.

${ }^{7}$ Richtungsweisend war Ralph-Rainer Wuthenows Aufsatz: Gefährdete Idylle (1966). Jetzt in: Jean Paul. Hrsg. von Uwe Schweikert. Darmstadt: Wissenschaftliche Buchgesellschaft 1974 (= Wege der Forschung, Bd. 336), S. 314-329; hier anknüpfend: Johannes Krogoll: Idylle und Idyllik bei Jean Paul. Eine Motivuntersuchung zur Rolle von Narrentum und Poesie im Werk des Dichters. Hamburg: o.V. 1972 sowie Jens Tismar: Gestörte Idyllen. Eine Studie zur Problematik der idyllischen Wunschvorstellungen am Beispiel von Jean Paul, Adalbert Stifter, Robert Walser und Thomas Bernhard. München: Hanser 1973 (= Literatur als Kunst).

${ }^{8}$ Vgl. Roger Ayrault: Jean Paul: Leben des vergnügten Schulmeisterlein Maria Wutz im Auenthal. Oder die Anfänge des Dichters Jean Paul. In: Interpretationen. Hrsg. von Jost Schillemeit. Bd.4: Deutsche Erzählungen von Wieland bis Kafka. Frankfurt a. M.: Fischer Taschenbuchverlag 1966 (=Fischer-Bücherei, Bd.721), S. 75-86; Kathleen Blake: What the narrator tells in Jean Paul's Wutz. In: German Quarterly 48, 1975, S.52-65; Hanns-Josef Ortheil: Idylle und Reflexion. Zur Geschichte von Jean Pauls Wutz. In: Literaturwissenschaftliches Jahrbuch N.F. 17 (1976), S. 83-97; Gonthier-Louis Fink: Der proteische Erzähler und die Leserorientierung in Jean Pauls Leben des vergnügten Schulmeisterlein Maria Wutz. In: Bild und Gedanke. Festschrift für Gerhart Baumann zum 60. Geburtstag. Hrsg. von Günter Schnitzler in Verbindung mit Gerhard Neumann und Jürgen Schröder. München: Fink 1980, S.271-287; Richard W. Hannah: The Tortures of the Idyll: Jean Pauls Wutz and the Loss of Presence. In: Germanic Review 56 (1981), S.121-127; Carl Pietzcker: „Mutter!”, sagt er zu seiner Frau, „ich fress” mich aber noch vor Liebe, Mutter!“ Oder Jean Paul bereitet uns mit seinem Leben des vergnügten Schulmeisterlein Maria Wutz ein bekömmliches Mahl. Wir dürfen uns selbst genießen. In: Freiburger literaturpsychologische Gespräche. Hrsg. von Johannes Cremerius u.a. Zweite Folge. Frankfurt a.M./Bern: Lang 1982 (=Literatur und Psychologie, Bd.10), S.49-97; Bernhard Böschenstein: Anmerkungen zur Metaphorik in Jean Pauls Leben des vergnügten Schulmeisterlein Maria Wutz in Auenthal. Elemente zu einem Kommentar. In Digressionen. Wege zur Aufklärung. Festgabe für Peter Michelsen. Hrsg. von Gotthard Frühsorge, Klaus Mangel, Friedrich Strack. Heidelberg: Winter 1984, S.149-175. 
In der bildenden Kunst ist die grundlegende Voraussetzung für die Auseinandersetzung mit Kunstwerken das allgemein akzeptierte Vorverständnis, das ein Kunstwerk vom Künstler als Artefakt exakt in der vom Betrachter exponierten Gestalt so gewollt sei. Dieser Kontrakt über die Intention des Autors, den wir als Museumsbesucher problemlos eingehen, fällt uns bei der Literatur offensichtlich weniger leicht. Setzten wir den selben Werkbegriff bei literarischen Texten an, hieße das doch, dass wir Unstimmigkeiten , die wir an der kompositorischen Textoberfläche oder bei der thematischen Durchführung sehen, als hermeneutische Probleme zu erkennen hätten, die wir mit dem Text haben. Dies hätte jedoch unter Umständen die Entwertung oder zumindest eine Umstrukturierung unseres Deutungsmusters zur Konsequenz. Gelingt die hermeneutische Operation nicht, dann ist es freilich einfacher, beim Autor Nachlässigkeiten oder 'Fehler' zu vermuten. Ein solcher Fall von Problemverschiebung auf den Autor scheint mir beim bisherigen Umgang der Forschung mit Jean Pauls Wutz vorzuliegen. Meine Überlegungen gehen von zwei zunächst vielleicht marginal erscheinenden „Blindstellen“ aus, die bisherige Deutungen nicht integriert haben, obwohl sie zwei basale Strukturmerkmale des Textes betreffen: Mündlichkeit und Komposition.

\section{Mündlichkeit}

Das Leben des vergnügten Schulmeisterlein Maria Wutz $^{9}$ ist der einzige Text von Jean Paul, der das komplexe dialogische Spiel zwischen Erzähler und Leser auch in einer Erzählsituation auf der Fiktionsebene abzubilden sucht und es damit verdoppelt. Schon der zweite Abschnitt der Erzählung versucht das, indem er die Lebensbeschreibung in eine mündliche Erzählsituation überführt:

Jetzt aber, meine Freunde, müssen vor allen Dingen die Stühle um den Ofen, der Schenktisch mit dem Trinkwasser an unsere Knie gerückt und die Vorhänge zugezogen und die Schlafmützen aufgesetzt werden, und an die grand monde über der Gasse drüben und ans Palais royal muß keiner von uns denken, bloß weil ich die ruhige Geschichte des vergnügten Schulmeisterlein erzähle - und du, mein lieber Christian, der du eine einatmende Brust für die einzigen feuerbeständigen Freuden des Lebens, die häuslichen, hast, setze dich auf den Arm des Großvaterstuhls, aus dem ich herauserzähle, und lehne dich zuweilen ein wenig an mich! Du machst mich gar nicht irre. (S. 422)

Jean Paul hat an der Vorstellung des mündlichen Erzählers, die in der Novellistik des Biedermeier und Realismus ein häufig verwandtes Gestaltungsmittel

\footnotetext{
${ }^{9}$ Im weiteren mit Seitenzahlen in Klammern zitiert nach: Jean Paul: Werke. Hrsg. von Norbert Miller. Bd.1. München/Wien: Hanser 1989.
} 
werden sollte, in seinem weiteren Werk nicht festgehalten, und selbst im Wutz lässt er seinen Erzähler die Fiktion einer häuslichen Erzählrunde am Feierabend ${ }^{10}$ nicht konsequent aufrechterhalten. Das Ende des Textes evoziert zwar noch einmal mündliches Erzählen und erinnert in einer abschließenden Rahmung an den geselligen Kreis zu Beginn („Es ist genug Freunde[...]“; S. 462), doch liegen dazwischen unspezifische Rezipienten-Adressen (S. 431, 448), die gleichwohl Hörern wie Lesern gehören können, und gegen Ende des Textes häufen sich irritierende Formulierungen, die jede Fiktion von Mündlichkeit unterlaufen.

Bemerkenswert ist nun, dass jene Abweichungen von der Fiktion der Mündlichkeit in der Forschung unwidersprochen immer noch als „Schönheitsfehler“11 gelten, die Jean Paul infolge einer Arbeitsunterbrechung unterlaufen seien, wie dies Eduards Berend 1927 formulierte. Berend nahm Hinweise im Text („so muß auf der Stelle abgebrochen werden, damit fortgerissen werde morgen um 6 Uhr“, S. 431) als lebenspraktische Anweisung ernst und ging deshalb davon aus, dass sich die Erzählung „auf drei Abende“12 verteilt. Unter dieser Perspektive, die dem Autor im Grunde mangelnde Kompetenz bei der Fiktionsbildung unterstellt, scheint „Schönheitsfehler“ noch eine wohlwollende Beurteilung. Gerade die Häufung der „Schönheitsfehler“ aber müsste doch Anlass zur Frage geben, warum Jean Paul seinen Erzähler in bewusster Störung der mündlichen Erzählfiktion von „Lesern“ (S. 446), „Fragezeichen“ (S. 447), „Gesetze[n] des Romans“ (S. 448), Druck (vgl. S. 451), „Postskript“ (S. 454) und „Seiten“ (S. 458) sprechen lässt und immer wieder vom Erzählen für Hörer zum Erzählen für Leser wechselt. Wieso weist der Erzähler immer wieder darauf hin, wie er gerade das Erzählmaterial anordnet (vgl. etwa S. 425)? Ein mündlicher Erzähler hätte wohl auch kaum Skrupel hinsichtlich der Authentizität des Erzählten, wie sie Wutz' Lebensbeschreiber beschäftigen. ${ }^{13}$ Zusammenfassend ist zu fragen: Warum lässt Jean Paul seinen Erzähler Mündlichkeit durchschaubar fingieren, statt sie stringent als literarische Fiktion aufrechtzuerhalten? Mir scheint diese Frage an Jean Pauls Text bisher zu Unrecht noch nicht gestellt worden zu sein.

\footnotetext{
${ }^{10}$ Jean Paul experimentiert im Wutz mit der mündlichen Erzählsituation, die freilich auch zeitgenössische Vorbilder hatte. Zu denken wäre u.a. an Joachim Heinrich Campes überaus erfolgreiche Bearbeitung von Daniel Defoes Robinson Crusoe für die Jugend; Joachim Heinrich Campe: Robinson der Jüngere, 2 Teile. Hamburg: Carl Ernst Bohn 1779/80. Campe hatte Defoes Roman von einer Ich-Erzählung in einen dialogisierten Text mit Zwischenfragen der Zuhörer umgeschrieben. Der Hausvater berichtet seinen Kindern an dreißig Erzählabenden die Geschichte von Robinson.

${ }^{11}$ Jean Pauls Sämtliche Werke. Historisch-kritische Ausgabe. Hrsg. von der Preußischen Akademie der Wissenschaften in Verbindung mit der Akademie zur wissenschaftlichen Erforschung und zur Pflege des Deutschtums und der Jean-Paul-Gesellschaft. 1.Abt 2.Bd.: Die unsichtbare Loge. Hrsg. von Eduard Berend. Weimar: Hermann Böhlaus Nachfolger 1927, S. LI.

12 Ebd.

13 „So beschreibt er wenigstens selber diese Erinnerung-hohen-Opern in seinen Rousseauschen Spaziergängen, die ich da vor mich lege, um nicht zu lügen...“ (S. 425).
} 


\section{Komposition}

Das Schulmeisterlein Wutz, gemessen an Jean Pauls späteren Erzähldimensionen ein eher schmales Erzählstück, erweist sich bei aller Plauderhaftigkeit des digressiven Erzählens als strenge Komposition. ${ }^{14}$ Nach dem Aufruf des Erzählgegenstandes, nämlich Wutz” „Leben und Sterben“ (S. 422f.) und der bereits beschriebenen Konstituierung einer mündlichen Erzählsituation in direkter Anrede an die Hörer (S. 422f.), beginnt der Erzähler, ganz wie es die Gattung der Lebensbeschreibung erwarten lässt, mit der chronologischen Abhandlung der Vita. Nach der Kindheit (S. 422) sind es drei exemplarische Lebensphasen, die der Erzähler mitteilt: Wutz in der Schulausbildung (S. 428-431), Wutz' Verliebtsein und Hochzeit (S. 431-454), Wutz' letzte Tage (S. 454-458) und sein Sterben (S. 459-461). In einer letzten Anrede, die wie zu Beginn der Erzählung erst Wutz und dann den `Zuhörern` der fingierten Runde gilt (S. 461f.), schließt sich der Erzählrahmen. Bei aller Lückenhaftigkeit der Darstellung (über Wutz' 43 Ehejahre etwa erfahren wir kein Wort) orientiert sich der Erzähler bei der Schilderung von Wutz' Leben doch stringent an dessen Abfolge. Signifikant scheint es deshalb, dass die Chronologie der Lebenserzählung an einer Stelle unterbrochen wird.

Der Erzähler unterbricht die Lebenschronologie zu Beginn des Textes nach der Schilderung der Kindheit. Einerseits will er auf Wutz' Erinnerungstechnik durch Verdunkeln und Schließen der Augen (S. 423) aufmerksam machen, insbesondere geht es ihm aber darum, auf den wichtige[n] Umstand“ hinzuweisen, „bei dem uns, wie man behauptet, so viel daran gelegen ist, ihn voraus zu hören“, nämlich den, „dass Wutz eine ganze Bibliothek - wie hätte der Mann sich eine kaufen können? - eigenhändig schrieb“ (S. 425). Wenn wir Jean Pauls Text also eine stringente Sinnkomposition zugrunde legen und ihn in seiner Abfolge nicht als Zufallsprodukt gelten lassen wollen, ist diese Abweichung vom chronologischen Erzählen zu begründen. Zu fragen wäre also, warum uns denn so viel daran gelegen sein sollte, über Wutz” „Erinnerung-hohen-Opern (S. 425) und seine selbstgeschriebene Bibliothek schon $\mathrm{zu}$ Beginn und an herausgehobener Stelle zu erfahren.

II.

Jeder, der einmal einen Roman von Jean Paul gelesen hat, weiß aus eigener Erfahrung, wie Jean Paul als Meisterschüler von Lawrence Sterne in seinen Texten immer wieder radikal alle Möglichkeiten des Erzähler-Leser-Dialogs

\footnotetext{
${ }^{14}$ Vgl. hierzu auch schon Roger Ayraults Beobachtungen; Roger Ayrault: Jean Paul: Leben des vergnügten Schulmeisterlein Maria Wutz in Auenthal (wie Anm.8), S.81; Ayraults Aufsatz fehlt allerdings die strikte Trennung von Erzähler und Autor.
} 
bemüht und seine Leser mit Digressionen und Extrablättern an die Grenzen ihrer Auffassungsgabe führt. Und so bekommen auch die 'Ungereimtheiten' dieses frühen Textes ${ }^{15}$ zunächst in einer naheliegenden reflexiven Lesart Plausibilität.

Die offensichtlich fingierte Mündlichkeit des Erzählens und die chronologische Abweichung werden bedeutsam, wenn das Schulmeisterlein Wutz als Text gelesen wird, der auf der Folie der Lebensdarstellung eines Sonderlings auch den Vorgang des Erzählens selbst verhandelt. Für das selbstreflexive Erzählerbild Jean Pauls stellt der Wutz einen Basistext dar. Konsens ist, dass Jean Paul in der Figur des Schulmeisterleins erstmals in seinem Werk den poetischen Schöpfungsakt in an Treffsicherheit kaum zu überbietender Bildlichkeit parodiert. $^{16}$

Innerhalb eines imaginären Korpus' den Vorgang des Erzählens reflektierender Texte scheint mir Jean Pauls Wutz jedoch noch einen besonderen Platz einzunehmen, den die Forschung kaum zur Kenntnis genommen hat. Im Wutz wird nicht nur in der Figur des Protagonisten über Schreiben reflektiert, sondern hier ist in Wechselwirkung der Ebenen Erzähler, Figur und Leser / Hörer an einem einzigen Text zu beobachten, mit welchen Mitteln Erzählen um 1790 möglich ist und wie über Erzählen reflektiert werden kann. Der Text ist also nicht nur selbstreflexiv, sondern reflektiert die Möglichkeiten narrativer Reflexivität, er ist - sozusagen eine Metareflexion, freilich eine fingierte mit narrativen Mitteln. Das Mittel, dessen sich der Erzähler bedient um die Metareflexion fingieren zu können, ist - so meine These - das Theater und seine Metaphorik. Theater ist nun freilich nicht auf einer inhaltlichen Ebene fiktionstragend, sondern theatrale Elemente gebündelt im Begriff der Inszenierung - werden zur ästhetischen Grundlage des Erzählens. Aus der beobachtenden Leserperspektive wird der Text dadurch zu einem komplexen Feld von narrativer Theatralität. Erst unter diesem Blickwinkel bekommen die „Unstimmigkeiten“, die der Ausgangspunkt meiner Überlegungen waren, tatsächlich Plausibilität und Sinn.

Lesen wir also die eingangs zitierte Szene einer häuslichen Erzählrunde einmal als theatrale Szene mit narrativen Mitteln. Die mündliche Erzählsituation, die sich so offensichtlich als solche durchschauen lässt, wird unter dieser Perspektive zu einer Erzählerinszenierung von oraler Geselligkeitskultur. Es geht also um mehr als um Theatermetaphorik, die bekanntlich Jean Pauls Texte durchzieht. ${ }^{17}$ Gezeigt werden soll, dass im Text wesentliche Elemente des The-

\footnotetext{
${ }^{15}$ Der Wutz gilt im allgemeinen als der erste nichtsatirische Text, den Jean Paul an der Wende vom Satiriker zum Romanschriftsteller schrieb. Zur Datierung des Textes auf Dezember 1790 vgl. Jean Pauls sämtliche Werke. Historisch-kritische Ausgabe. 1. Abt. 2. Bd. (wie Anm. 11), S. XLVIII.

${ }^{16}$ Vgl. hierzu auch Roger Ayrault: Jean Paul: Leben des vergnügten Schulmeisterlein Maria Wutz in Auenthal (wie Anm.8), S.85.

${ }^{17}$ Vgl. etwa Bernhard Böschenstein: Jean Pauls Romankonzeption (1968). Jetzt in: Jean Paul. Hrsg. von Uwe Schweikert (wie Anm.7), S.330-352, hier: S.349. Zuletzt und mit Nachweis
} 
aters narrativ erzeugt werden: In der Evokation einer regelrechten Bühnenszene (S. 422) wird vor uns Lesern ein Publikum (,jetzt aber, meine Freunde“) und der Ort des Erzählens („der Großvaterstuhl, aus dem ich herauserzähle“) konstituiert. Ausgangspunkt der Erzählung ist also die szenisch vergegenwärtigte Fokussierung auf einen Erzähler. Erzeugt wird dies (wie im Theater) durch Bündelung und Selektion der Wahrnehmung. „Jetzt aber, meine Freunde, müssen vor allen Dingen die die Stühle um den Ofen, der Schenktisch mit dem Trinkwasser an unsere Knie gerückt und die Vorhänge zugezogen und die Schlafmützen aufgesetzt werden“. Mit dem Schließen der Vorhänge wird ein „Zuschauerraum geschaffen, zudem hilft die Verdunkelung dabei, abschweifende Gedanken an die an die ausgegrenzte Öffentlichkeit zu unterbinden (,an die grand monde über der Gasse drüben und ans Palais royal muss keiner von uns denken."). Doch diese Szene am Beginn des Textes ist mehr als ein geschickter Erzählanfang, korrespondieren die Elemente der Inszenierung doch mit Wutz’ eigener Erinnerungsmethode, mit denen er sich in den „ältern Jahren“ seine Kindheit wieder ins Bewusstsein holt:

Im Dezember von jenen ließ er allemal das Licht eine Stunde später bringen, weil er in dieser Stunde seine Kindheit - jeden Tag nahm er einen andern Tag vor rekapitulierte. Indem der Wind seine Fenster mit Schnee-Vorhängen verfinsterte und indem ihn aus den Ofen-Fugen das Feuer anblinkte: drückte er die Augen zu und ließ auf die gefrornen Wiesen den längst vermoderten Frühling niedertauen [...]. (S. 423)

Wutz` „palingenesierende Winter-Abendstunde“ (S. 424) und die Basisfiktion der Erzählsituation funktionieren offensichtlich gleichermaßen über Wahrnehmungsselektion und Wahrnehmungsfokussierung nach theatralem Muster. ${ }^{18}$ Hier Konzentration auf Erinnerung der eigenen Geschichte, dort Konzentration auf Erzählung gewordene erinnerte Geschichte. Aus der Beobachterperspektive hierarchisieren sich die Inszenierungsebenen in vorgelagerte SelbstInszenierungen von Wutz und darauf aufbauende Erzähler-Inszenierungen.

Bleiben wir zunächst bei Wutz' Selbstinszenierungen. Während Wutz die Erinnerung an die Kindheit über das Schließen der Augen hervorholt - also

der Forschung zur Theatermetaphorik im Werk Jean Pauls seit Max Kommerell siehe Monika Schmitz-Emans: Dramatische Welten und verschachtelte Spiele. Zur Modellfunktion des Theatralischen in Jean Pauls Romanen. In: Jahrbuch der Jean-Paul-Gesellschaft 22 (1987), S. 67-93, hier: S. 70f. Ausgehend von Theater als Thema in den Texten Jean Pauls versteht Schmitz-Emans unter ,Theatralischem' Analogiebildungen zwischen Leben und Schauspiel, also Theatralisierungen des Lebens, die etwa die Problematik ,Schein und Sein' thematisieren. Sie liest den Titan als eine Variation des barocken Welttheater-Konzepts, dessen Wirklichkeit sich aber nicht nur als „theatralische Inszenierung“, sondern durch die „Erfahrung einer Pluralität konkurrierender Wirklichkeits-Entwürfe präsentiere“(S.73).

${ }^{18}$ So fehlt bei Wutz auch nicht der Rückholeffekt in die 'Wirklichkeit' durch die Aufhebung der Verdunklung. „Und alle Jahre, sooft er diese Retourfuhre seiner Kindheit [...] veranstaltete, vergaß uns erstaunt' er - sobald das Licht angezündet wurde - dass in der Stube, die er sich wie ein Loretto-Häuschen aus dem Kindheit-Kanaan herüberholte, er ja gerade jetzt säße“ (S. 425, Kursivierung M.H.) 
Konzentration nach innen durch weitgehenden Ausschluss der Sinne - wendet er in den letzten Tagen, als er schon ans Bett gefesselt ist, eine gegensätzliche Methode an: Erinnerung durch intensives Sehen und Fühlen. Mit „Rudera und Spätlinge[n] seiner verspielten Kindheit (S. 455), Erinnerungszeichen wie eine grüntaftene Kinderhaube, eine Kinderpeitsche, ein Fingerring von Zinn, ein beschmutztes Schreibbuch (vgl. ebd.) errichtet Wutz auf der Bettdecke den „Antikentempel“ (ebd.) seiner Kindheit, durch den er zusammen mit dem bei ihm sitzenden Erzähler flaniert. Seine „größte Krankenlabung“ ist jedoch „ein alter Kalender“[...] und die abscheulichen 12 Monatskupfer desselben“ (S. 458). Er durchwanderte nämlich die 11 Monat-Vignetten - die des Monats, worin er wanderte, ließ er weg - und phantasierte in die Holzschnitt-Auftritte alles hinein, was er und sie nötig hatten“ (S. 458, Kursivierung M.H.). Die Monatskupfer eines Kalenders werden zu Bühnenbildern und dienen Wutz als Erlebnisraum für Spaziergänge seiner Phantasie.

Auf die Erinnerungsinszenierungen folgen Wutz' Selbstinszenierungen in und durch seine Texte, die der Erzähler in der Bibliothek vorfindet und die „seine Lebensbeschreiber fast nur abzuschreiben brauchen“ (S. 433). Ich greife eine besonders markante Szene heraus. Sie stammt aus „Werthers Freuden“ und zeigt einerseits, wie Wutz durch selektive Wahrnehmung die Umwelt zum Spiegel seiner Innenwelt umfunktioniert, bis beide wie „zwei Muschelschalen“ (S. 435) kaum noch zu unterscheiden sind. ${ }^{19}$ Mehr noch scheint mir folgende Szene aber zu belegen, wie Wutz seine Identität, das heißt hier seine Gefühlsseligkeit, erst aus einer, nach seinen Vorstellungen zur theatralen Inszenierung funktionalisierten Umwelt gewinnt:

Er ging da sonntags nach der Abendkirche heim nach Auenthal und hatte mit den Leuten in allen Gassen Mitleiden, dass sie daheim bleiben mußten. Draußen dehnte sich seine Brust mit dem aufgebaueten Himmel vor ihm aus, und halbtrunken im Konzertsaal aller Vögel horcht' er doppelseitig bald auf die gefiederten Sopranisten, bald auf seine Phantasien. [...] Da er immer kurz vor und nach SonnenUntergang ein gewisses wollüstiges trunkenes Sehnen empfunden hatte - die Nacht aber macht wie ein längerer Tod den Menschen erhaben und nimmt ihm die Erde -: so zauderte er mit seiner Landung im Auenthal so lang', bis die zerfließende Sonne durch die letzten Kornfelder vor dem Dorf mit Goldfäden, die sie gerade über die Ähren zog, sein blaues Röckchen stickte und bis sein Schatten an den Berg über den Fluß wie ein Riese wandelte. Dann schwankte er unter dem wie aus der Vergangenheit herüberklingenden Abendläuten ins Dorf hinein und war allen Menschen gut, selbst dem Präfektus. (S. 434, Kursivierung M.H.)

Wutz wartet den günstigsten Moment ab, bis alle theaterpraktischen Voraussetzungen für seine Selbstinszenierung gegeben sind: Die Abendsonne benützt er als Scheinwerfer, die seinen blauen Rock vergoldet und so die Kleidung zum Theaterkostüm macht, nämlich zu Werthers blauem Rock mit gelber Weste. Die Lichtregie ermöglicht ihm dabei, sich selbst übersteigert groß und gleich-

${ }^{19}$ Vgl. etwa Hanns-Josef Ortheil: Idylle und Reflexion (wie Anm.8), S.90f. 
zeitig in der Landschaft zerfließend wahrzunehmen. Wutz erlebt seine Umgebung, einschließlich des Glockenläutens, das ihm die musikalische Einstimmung und Untermalung liefert, nur als gigantische Theaterkulisse. Jene kompromisslose Identitäts- und Weltkonstruktion über das eigene Ich, die Wutz zu eigen ist, führt schließlich zurück zum Thema 'Schreiben' und dem „wichtigen Umstand“, den der Erzähler so deutlich betont.

Der Erzähler führt Wutz' selbstgeschriebene Bibliothek bereits zu Beginn der Lebensbeschreibung gegen die Chronologie ein, weil er Wutz' ,Marotte’ als Folie braucht, vor der er dann seine eigenen Reflexionen über Schreiben und Schreibprobleme entwickeln kann. Wutz ist das ins Maßlose gesteigerte Abbild eines von Produktionssorgen freien Schreibers. Bei aller Liebenswürdigkeit ist er dennoch nicht mehr als die (Selbst)Inszenierung eines Schriftstellers, den - wie der Erzähler sehr wohl weiß - letztlich nur so viel verbindet wie einen Soldaten auf der Bühne mit einem Soldaten im Zuschauerraum.

Sein Schreibzeug war seine Taschendruckerei; jedes neue Messprodukt, dessen Titel das Meisterlein ansichtig wurde, war nun so gut als wie geschrieben oder gekauft: denn er setzte sich sogleich hin und machte das Produkt und schenkt' es seiner ansehnlichen Büchersammlung [...]Nur ein Buch ließ er in sein Haus, den Messkatalog [...]. [...] abends musste der gute Mann nach dem Abendessen noch gar um den Südpol rudern und konnte auf seiner Cookischen Reise kaum drei gescheite Worte zum Sohne nach Deutschland hinaufreden. Denn da unser Enzyklopädist nie das innere Afrika oder nur den spanischen Maulesel-Stall betreten, oder die Einwohner von beiden gesprochen hatte: so hatt' er desto mehr Zeit und Fähigkeit, von beiden und allen Ländern reichhaltige Reisebeschreibungen zu liefern - [...] Wutz zerrete sein Reisejournal auch aus niemand anders als aus sich. (S. $425 \mathrm{ff}$.

Jenseits von Ironie und handfester Parodie auf den Geniekult lässt sich Wutz als autopoietische Künstlerfigur lesen. Wutz zeugt aus sich selbst Kunst, Erfahrung und Erkenntnis und dient deshalb als Ausgangspunkt für eine Diskussion von Authentizitäts- und Imaginationsproblemen, die den Erzähler selbst beschäftigen. Daran erinnert sei übrigens, dass der Erzähler Wutz nur kennen lernt und die Aufgabe der Lebensbeschreibung übertragen bekommt, weil er auch ein „Büchermacher“ (S. 454) ist. Im Gegensatz zu Wutz, der in einem einzigartigen zirkulären Erkenntnisprozess sogar beim Studium selbstverfasster Freimaurerreden immer tiefer in die Geheimwissenschaft eindringt (vgl. S. 428), gelingt dem Erzähler die Selbsterzeugung von Wissen jedoch nicht so leicht, er hat vielmehr ein handfestes Problem: Der Erzähler muss eine Hochzeit beschreiben, ohne selbst eine erlebt zu haben (vgl. S. 446). Wutz konfrontiert so den Erzähler mit Grundfragen des Schreibens. Wie kann ich über etwas schreiben, worüber ich eigentlich nicht schreiben kann, weil ich es nicht aus eigener Erfahrung kenne. Die Auflösung des Problems und die Einübung in das Wechselverhältnis von Text und Leben erfährt der Erzähler bei der Durchführung seiner Aufgabe: Nämlich Wutz „Lebensbeschreibung, die in der ganzen 
Bibliothek wäre“ (S. 454) aufzudecken, das Leben aus dem Text wieder herauszudestillieren und zugleich umgekehrt Leben (z.B. eine Hochzeit) zu Literatur zu machen.

Während Wutz naiv glaubt, sein Leben in Text überführen zu können, überführt der Erzähler einen Text in einen anderen Text. Wutz und sein Erzähler verlebendigen in gewisser Weise Schillers spätere Kategorien ,naiv’ und ,sentimentalisch'. Der Erzähler weiß um die Differenz zwischen Text und Leben und verkörpert als sentimentalischer Dichter selbst die Entstehung von Literatur aus Literatur, wenn er den Stoff seiner Literatur aus Büchern holt, die er beim Erzählen vor sich liegen hat, „um nicht zu lügen“ (S. 425). Wir Leser werden Zeugen, wie der Erzähler anhand der in Wutz' Texten inszenierten Lebensgeschichten dessen Vita entwickelt und sehen ihn am Ende vor seiner größten Bewährungsprobe: Wutz' Tod.

„Wie war dein Leben so sanft und meerstille, du vergnügtes Schulmeisterlein Wutz!“ (S. 422) Schon der erste Satz exponiert das Thema Tod, das sich durch die ganze Erzählung als Subtext ziehen wird. ${ }^{20}$ In einem Punkte allerdings unterscheidet sich diese Aufgabe wesentlich von der bisherigen Lebensbeschreibung: Für Wutz' Tod gibt es keine Textvorlage mehr. Wutz' Tod hat der Erzähler leibhaftig und 'authentisch' erlebt. Der Tod wäre also nach den vielen kleinen Lebensinszenierungen in Wutz' Texten die einzige Phase im Lebenslauf, die der Erzähler aus eigener Wahrnehmung schildern kann. Doch es zeigt sich, auch das persönliche Erleben garantiert im Moment der Verschriftlichung keinen authentischen Text. Die Erzählung von Wutz' Tod gleicht sich Wutz' eigenen Lebensbeschreibungen vielmehr in der Darstellungsmethode an. Denn seine narrative Aufgabe wird auch der Erzähler in Form einer Inszenierung bewältigen: Als „Leidenstheater“ (S. 461) im Mondlicht mit Uhrenmetaphorik und personifiziertem Tod. Wir Leser werden zu Beobachtern der „überirdischen Szene“ (ebd.):

Ich war allein in der Stube - ich hörte nichts als den Atemzug des Kranken und den Schlag meiner Uhr, die sein kurzes Leben wegmaß - der gelbe Vollmond hing tief und groß in Süden und bereifte mit seinem Totenlichte die Maiblümchen des Mannes und die stockende Wanduhr und die grüne Haube des Kindes - [...] - Am stillen Himmel wurde zuweilen eine fackelnde Sternschnuppe niedergeworfen, und sie verging wie ein Mensch - [...] - Ich sah, dass der, dem damals dieser Kirschbaum Wohlgeruch und Träume gab, dort im drückenden Träume geruchlos liege und vielleicht noch heute aus dieser Stube ausziehe und das alles, alles vor-

\footnotetext{
${ }^{20}$ Neben den permanenten Anspielungen scheint besonders signifikant, dass der Text auch in Augenblicken höchster Empfindung und Lust auf die Nähe zum Tod verweist. Vgl. Wutz Ausflug mit Justine, in der wiederum die Abendsonne als gefühlsstimulierende Beleuchtungsmaschinerie fungiert. „Die Sonne kroch jetzt ein zu einem einzigen roten Strahle, der mit dem Widerschein der Abendröte auf dem Gesicht der Braut zusammenkam; [...] und es war ihm als läutete die Abendglocke die Welt und noch einmal seinen Vater zur Ruhe - und zum ersten und letzten Male in seinem Leben stieg sein Herz über die irdische Szene [!] hinaus - und es rief, schien ihm, etwas von den Abendtönen herunter, er werde jetzo vor Vergnügen sterben...“ (S. 444f.).
} 
über sei und niemals wiederkomme... und in dieser Minute fing Wutz mit dem ungelähmten Arm nach etwas, als wollt' er einen entfallenden Himmel erfassen - und in dieser zitternden Minute knisterte der Monatszeiger meiner Uhr und fuhr, weil's 12 Uhr war vom 12ten Mai zum 13ten über...Der Tod schien mir meine Uhr zu stellen, ich hörte ihn den Menschen und seine Freuden käuen, und die Welt und die Zeit schien in einem Strom von Moder sich in den Abgrund hinabzubröckeln!...(S. 459f.).

Was bleibt, sind theatrale Inszenierungen, die über die Metaphorik genügend Distanz erzeugen und Schreiben auch im Bewusstsein des Todes möglich machen. Bemerkenswert ist: Selbst der persönlich miterlebte Tod bringt keine anderen Darstellungsmittel hervor als Wutz' phantastische Kopfgeburten und erhält dadurch im Text denselben Realitätsstatus. Ein inszeniertes Leben schließt mit einem inszenierten Tod. Verwundern sollte es eigentlich nicht bei einem Erzähler, der weiß, dass alles, was er entfaltet, „von fremden oder eigenen Fingern bloß - gemalt [ist] mit Dinte oder Druckerschwärze“ (S. 446) und der angesichts einer ihn total umstellenden virtuellen Welt dennoch unbeirrbar am Erzählen derselben festhält, um wenigstens sich selbst „eine Lustpartie zusammen [zu] machen“ (S. 446).

III.

Ich breche hier ab, nicht jedoch ohne zumindest skizzenhaft meine Überlegungen zum Wutz auch mit dessen Schöpfer, dem Autor Jean Paul selbst, in Verrechnung gebracht zu haben; wird der Wutz doch allgemein mit Jean Pauls so genanntem Todeserlebnis verbunden, von dem das Tagebuch vom 15. November 1790 berichtet:

Wichtigster Abend meines Lebens: denn ich empfand den Gedanken des Todes, dass es schlechterdings kein Unterschied ist, ob ich morgen oder in 30 Jahren sterbe, dass alle Plane und alles mir davonschwindet und dass ich die armen Menschen lieben sol, die sobald mit ihren Bisgen Leben niedersinken - der Gedanke ging bis zur Gleichgültigkeit an allen Geschäften ${ }^{21}$.

Der Wutz führe als „unmittelbare Frucht dieses Erlebnisses“ die „neugewonnene Einheit von Leben und Tod“ ${ }^{22}$ vor. Nun ist der Tagebucheintrag, wie wir heute wissen, nicht der einzige Text Jean Pauls aus diesen Monaten, der sich mit dem Tod beschäftigt. Zusammen mit „Meine Überzeugung, dass ich todt bin“, einem satirischen Text von 1789 und einem Text aus der Sammlung „Dichtung. November 1790“ wird der Tagebucheintrag wie der Wutz zum Glied einer Textkette, die Verschriftlichungsstrategien aufweist. Götz Müller, der den Text aus „Dichtung. November 1790“ erstmals veröffentlichte, hat -

\footnotetext{
${ }^{21}$ Zitiert nach: Uwe Schweikert: Jean Paul. Stuttgart: Metzler 1970, S.26 (= Sammlung Metzler, Bd.91).

${ }^{22}$ Ebd.
} 
allerdings ohne den Wutz in seine Überlegungen einzubinden - auf den Perspektivenwechsel hingewiesen, der sich in den Texten vollzieht. Die Veränderung der Dichtung gegenüber dem Tagebuch ist „die Vorstellung, sich selbst als Toter zu sehen“²3. Diese Wahrnehmungsverschiebung vom persönlichen existenziellen Erleben zum literarisch erzeugten Tod aus der Beobachterperspektive wiederum ist in diesem Text die Voraussetzung für die „theatralische Installation“‘24 eines Sylvesterabends zu fünft, den Jean Paul unter das Motto stellt: „wir wollen alle einander tod sehen“ ${ }^{25}$.

Jean Paul, der jedem Menschen einen 15.November wünschte, arbeitet an der Erfüllung dieses Wunsches, indem er seine existentielle Erfahrung als inszenierten Tod in Texten weitergibt. Wie der Erzähler von Wutz' Leben weiß, dass das Leben vergebens in Texten zu suchen ist, denn im Text finden sich nur immer wieder andere Texte, so gilt auch für Jean Paul: Der Tod ist nur als Text zu fassen - und verschwindet dabei aber gleichzeitig. Um dies darstellen zu können und für Leser mittelbar zu machen, setzt der Text Strukturen ein, die auf das Theater zurückgreifen, narrative Theatralität erzeugen. Eine existenzielle Erfahrung jenseits des Textes und dessen sprachlichen Materials freilich ist damit nicht zu greifen.

Mit der Exposition dieser Problematik bezieht Jean Paul schon 1790 im Wutz eine Gegenposition zum lebendigen Dichterglauben wie ihn die deutsche Klassik mit Goethe, Schiller und Herder verkörpert. Wutz lässt sich als anrührende Idylle und gleichzeitig als hochartifizieller Text lesen, der die Bedingungen der Möglichkeit von Kunst grundsätzlich in Frage stellt. Deshalb ist diese kleine Erzählung auch ein wichtiges Glied in der langen Kette der literarischen Tode und Scheintode im Werk Jean Pauls. Von hieraus - so schiene mir - wäre in einem weiteren Schritt die in der Jean-Paul-Forschung längst überfällige Integration des Wutz aus dem Schattendasein eines „Anhängsels“ ${ }^{26}$ in den Werkkontext der Unsichtbaren Loge zu leisten. Die Art und Weise wie im Le-

${ }^{23}$ Götz Müller: „Ich vergesse den 15. November nie“. Intertextualität und Mehrfachbesetzung bei Jean Paul. In: Die Genese literarischer Texte. Modelle und Analysen. Hrsg. von Axel Gellhaus zusammen mit Winfried Eckel u.a. Gedenkschrift für Beda Allemannn. Würzburg: Königshausen \& Neumann 1994, S. 57-75, hier: S. 61.

${ }^{24}$ Ebd., S. 62.

${ }^{25}$ Götz Müllers wegweisender Aufsatz entwickelt aus weiteren Texten dann plausibel den engen Zusammenhang mit Jean Pauls Lebensthema Scheintod als reine Kontextverschiebung: „Aus dem Imperativ Wir-wollen-einander-tod-sehen wird das epische Präteritum Als-ich-totzu-sein-schien.“ Ebd., S. 66

${ }^{26}$ Symptomatisch hierfür erscheint die Selbstverständlichkeit mit der Klaus Pauler in seiner Textausgabe der Unsichtbaren Loge nach der Erstausgabe 1793 den der Loge „aufgepfropfte[n]“ Wutz aus Kostengründen nicht abdruckt. Vgl. hierzu Jean Paul: Die Unsichtbare Loge. Text der Erstausgabe von 1793 mit den Varianten der Ausgabe von 1826, Erläuterungen, Anmerkungen und Register. Hrsg. von Klaus Pauler. München: Text + Kritik 1981, S. 211. Erste Ansätze zu einer Neubewertung des Wutz im Sinne einer „Komplementärwelt“ zur Loge bei Norbert Miller: Die Unsterblichkeit der zweiten Welt. Jean Pauls literarische Anfänge und die Entstehung seiner Romanwelt. In: Jean Paul: Sämtliche Werke. 2.Abt. 4.Bd.: Jugendwerke und vermischte Schriften. Hrsg. von Norbert Miller und Wilhelm SchmidtBiggemann. München/Wien: Hanser 1985, S. 9-85, hier: S. 80 
ben des vergnügten Schulmeisterlein Maria Wutz das Thema Schreiben, Leben und Sterben auf der Bühne des Textes in narrativen Inszenierungen verhandelt wird, das macht den Wutz nicht nur zum Kern der Unsichtbaren Loge, sondern rückt ihn vielmehr in den Mittelpunkt von Jean Pauls Werk. 